\title{
SARS-CoV-2 Vaccine Responses in Individuals with Antibody Deficiency: Findings From The COV-AD Study
}

Adrian M Shields ( a.m.shields@bham.ac.uk)

University of Birmingham https://orcid.org/0000-0001-5345-2156

Sian E. Faustini

University of Birmingham

Harriet J. Hill

University of Birmingham

Saly Al-Taei

University of Birmingham

Chloe Tanner

University of Birmingham

Fiona Ashford

University of Birmingham

Sarita Workman

Department of Immunology, Royal Free London NHS Foundation Trust

Fernando Moreira

Department of Immunology, Royal Free London NHS Foundation Trust

Nisha Verma

Department of Immunology, Royal Free London NHS Foundation Trust

Hollie Wagg

University of Birmingham

Gail Heritage

University of Birmingham

Naomi Campton

University of Birmingham

Zania Stamataki

University of Birmingham

Paul Klenerman

University of Oxford

Sarah Goddard

University Hospitals North Midlands

Sarah Johnston

Department of Clinical Immunology, North Bristol NHS Trust

Aarnoud Huissoon

University Hospitals Birmingham NHS Foundation Trust

Claire Bethune

University Hospitals Plymouth NHS Trust

\section{Suzanne Elcombe}

Department of Allergy and Clinical Immunology, Newcastle upon Tyne Hospitals NHS Foundation Trust

David M. Lowe

Department of Immunology, Royal Free London NHS Foundation Trust

Smita Y. Patel

University of Oxford

Sinisa Savic

Department of Allergy and Clinical Immunology, Leeds Teaching Hospitals NHS Trust

Siobhan O. Burns

Department of Immunology, Royal Free London NHS Foundation Trust

Alex G. Richter

University of Birmingham

COV-AD Consortium

on behalf of the COV-AD consortium 


\section{Research Article}

Keywords: COVID-19, CVID, Inborn errors of immunity, primary immunodeficiency, secondary immunodeficiency, vaccination, SARS-CoV-2

Posted Date: January 4th, 2022

DOI: https://doi.org/10.21203/rs.3.rs-1180392/v1

License: (c) (i) This work is licensed under a Creative Commons Attribution 4.0 International License. Read Full License

Version of Record: A version of this preprint was published at Journal of Clinical Immunology on April 14th, 2022. See the published version at https://doi.org/10.1007/s10875-022-01231-7. 


\section{Abstract \\ Background}

Vaccination prevents severe morbidity and mortality from COVID-19 in the general population. The immunogenicity and efficacy of SARS-CoV-2 vaccines in patients with antibody deficiency is poorly understood.

\section{Objectives}

COVID in patients with antibody deficiency (COV-AD) is a multi-site United Kingdom study that aims to determine the immune response to SARS-CoV-2 infection and vaccination in patients with primary or secondary antibody deficiency, a population that suffers from severe and recurrent infection and does not respond well to vaccination.

\section{Methods}

Individuals on immunoglobulin replacement therapy or with an IgG less than $4 \mathrm{~g} / \mathrm{L}$ receiving antibiotic prophylaxis were recruited from April 2021 . Serological and cellular responses were determined using ELISA, live-virus neutralisation and interferon gamma release assays. SARS-CoV-2 infection and clearance were determined by PCR from serial nasopharyngeal swabs.

\section{Results}

$5.6 \%(n=320)$ of the cohort reported prior SARS-CoV-2 infection, but only $0.3 \%$ remained PCR positive on study entry. Seropositivity, following two doses of SARS-CoV-2 vaccination, was $54.8 \%(n=168)$ compared with $100 \%$ of healthy controls $(n=205)$. The magnitude of the antibody response and its neutralising capacity were both significantly reduced compared to controls. Participants vaccinated with the Pfizer/BioNTech vaccine were more likely to be seropositive ( $65.7 \%$ vs $48.0 \%, p=0.03$ ) and have higher antibody levels compared with the AstraZeneca vaccine (IgGAM ratio 3.73 vs 2.39 , $p=0.0003$ ). $T$ cell responses post vaccination were demonstrable in $46.2 \%$ of participants, were associated with better antibody responses but there was no difference between the two vaccines. Eleven vaccine-breakthrough infections have occurred to date, 10 of them in recipients of the AstraZeneca vaccine.

\section{Conclusion}

SARS-CoV-2 vaccines demonstrate reduced immunogenicity in patients with antibody deficiency with evidence of vaccine breakthrough infection.

\section{Introduction}

The immunological correlates of protection against SARS-CoV-2 infection and severe COVID-19 are not yet known. The passive acquisition [1] or development of anti-SARS-CoV-2 spike glycoprotein antibodies following infection [2-4] confers significant protection against future disease and, in some cases, facilitates viral clearance in individuals that fail to mount effective immune responses following infection [5-9].

Vaccination against SARS-CoV-2 is the most effective public health intervention to prevent severe morbidity and mortality from COVID-19 in the general population [10-12]. A meta-analysis of vaccine efficacy studies has suggested that neutralising antibody levels are strongly associated with protection from symptomatic infection [13]. However, it is well recognized that patients with immunodeficiency may not respond optimally to vaccination. To date, SARS-CoV2 vaccine immunogenicity and efficacy has not been comprehensively studied in individuals with primary and secondary immunodeficiency; preliminary studies suggest seropositivity rates following vaccination vary between $20.0-83.0 \%$ [14-18]. Given the significantly increased risk of morbidity and mortality from COVID-19 that these patients face $[19,20]$, understanding the immunogenicity and efficacy of vaccines in this population is of critical importance.

COVID in patients with antibody deficiency (COV-AD) is a multi-site United Kingdom study that aims to: i) determine the prevalence of asymptomatic and symptomatic SARS-CoV-2 infection in patients with primary and secondary antibody deficiency, ii) determine how frequently SARS-CoV-2 viral persistence occurs in patients with primary and secondary antibody deficiency and iii) characterise the immune response of these patients following SARS-CoV-2 infection and vaccination. This manuscript presents an interim analysis of 320 participants in the COV-AD study to describe responses to the primary course of vaccination and the risk of vaccine breakthrough and viral persistence.

\section{Methods}

\section{Patient eligibility and recruitment}

From March 2021, patients with primary or secondary antibody deficiency were recruited from the following Immunology centres across the United Kingdom: University Hospitals Birmingham NHS Foundation Trust, Royal Free London NHS Foundation Trust, North Bristol NHS Trust, Oxford University Hospitals NHS Foundation Trust, Leeds Teaching Hospitals NHS Trust, University Hospitals North Midlands NHS Trust, University Hospitals Plymouth NHS Trust, Newcastle Upon Tune Hospitals NHS Foundation Trust. 
Patients were eligible for study entry if: i) they were over 18 years of age and ii) they were receiving immunoglobulin replacement therapy or they had a serum IgG concentration less than $4 \mathrm{~g} / \mathrm{L}$ and were receiving regular antibiotic prophylaxis to prevent infections. Participants' underlying immunological diagnosis was made according the European Society of Immunodeficiency Clinical Working Party criteria. In this manuscript, "other primary antibody deficiency" has been used to encompassing individuals who do not fulfil the diagnostic criteria for CVID, XLA or any monogenic immunodeficiency but are still believed to have a primary humoral immunodeficiency.

At study entry, meta-data including demographics, immunological diagnosis and immunological parameters (e.g. baseline IgG concentration, trough IgG concentration, lymphocyte enumeration and whether an individual had previously tested positive for SARS-CoV-2 by PCR) were documented. All participants submitted a postal nasopharyngeal swab to determine SARS-CoV-2 status by PCR as previously described [21]. Individuals with a positive SARS-CoV-2 PCR were sent follow-up swabs at two-weekly intervals until a negative swab was returned. Results of routine clinical swabs were also documented as part of this study.

Study participants were then followed longitudinally through the United Kingdom routine SARS-CoV-2 vaccination schedule. Participants received two doses of either the AstraZeneca ChAdOx1 nCoV-19 (Vaxzevria) or the Pfizer BioNTech 162b (Tozinameran) vaccine according to the extended vaccine schedule mandated by the UK Chief Medical Officers (https://www.gov.uk/government/publications/prioritising-the-first-covid-19-vaccine-dose-jcvistatement/optimising-the-covid-19-vaccination-programme-for-maximum-short-term-impact).

A cohort of 205 healthy control participants were recruited from the COVID-19 Convalescent (COCO) study. These participants were otherwise healthy health care workers (median age 44 years, (range 22-66 years), 28\% male), vaccinated with two doses of Pfizer BioNTech 162b on the extended UK dosing schedule and sampled 1-2 month after vaccination.

Participants were sampled, whenever possible, prior to their second vaccine dose and between 1 and 2 months following their second vaccine dose. When this was not possible, a single sample was taken at no fixed time point following their second vaccine dose.. To facilitate sampling, individuals were given the option of remote sampling by dried blood spot (DBS) or for an enhanced cohort venous blood sampling to enable cellular analysis. We have previously recorded excellent concordance between serum and DBS samples using this assay [22]. Serum or dried blood samples [22] were tested for the presence of anti-spike glycoprotein antibodies (The Binding Site, Birmingham, UK). Results are reported as an IgGAM ratio (optical density compared with calibrator) and results $>1.0$ are defined as seropositive. The ratio provides a semi-quantitative assessment of the magnitude of the antibody responses [23]. Serum samples were also assessed for neutralising capacity using an in-house live virus neutralisation assay. T cell responses were assessed using the T-SPOT ${ }^{\circledR}$.COVID assay (Oxford Immunotec, Abingdon, UK), an ELISPOT based IFN-gamma release assays utilising peptide pools derived from the SARS-CoV-2 spike and nucleocapsid proteins; 0-4 spots per well is considered negative, 5-7 spots per cell, borderline and greater than 7 spots per well a positive response. Detailed descriptions of the methods are available in the Supplementary Methods.

\section{Statistical analysis}

Data were analysed using Graph Pad Prism 9.0 (GraphPad Software, San Diego, California USA). Continuous variables were analysed using the 2-tailed MannWitney $\mathrm{U}$ test, categorical variables analysed using the $\chi 2$ test and the relationship between antibody response, time and vaccine received by 2 -way ANOVA. Spearman's rank correlation coefficient was used to assess the relationship between antibody concentrations and neutralisation potential.

\section{Ethical approval and funding}

This study was approved by the London - Dulwich Research Ethics Committee (REC reference: 21/LO/0162) and funded by United Kingdom Research and Innovation (MR/W002663/1). Serological responses from healthy individuals are from participants recruited to the COVID-19 Convalescent (COCO) immunity study (REC reference 20/HRA/1817). All participants provided written informed consent prior to participation in this study.

\section{Results}

The results of 320 participants in the COV-AD study are available for interim analysis (Table 1). The median age of participants was 58.5 years and $40 \%$ ( $n=128 / 320)$ were male. The median interval between the first and second vaccine dose was 76 days; $42.1 \%(n=135 / 320)$ of participants received the Pfizer BioNTech 162b vaccine and 55.0\% $(n=176 / 320)$ the AstraZeneca ChAdOx1 nCoV-19 vaccine. 


\begin{tabular}{|c|c|}
\hline $\mathbf{N}$ & 320 \\
\hline \multirow[t]{2}{*}{ Age $(y r)$} & 58.5 \\
\hline & $(43.0-68.8)$ \\
\hline Sex (male) n, \% & $128(40.0)$ \\
\hline Vaccination & $135(42.1)$ \\
\hline - Pfizer BioNTech 162b2 & $176(55.0)$ \\
\hline - AstraZeneca ChAdOx1 nCoV-19 & $2(0.63)$ \\
\hline - Unvaccinated & $7(2.2)$ \\
\hline \multicolumn{2}{|l|}{ - Unknown } \\
\hline Vaccine dosing interval (d) & $76(70-78)$ \\
\hline Diagnosis & $139(43.4)$ \\
\hline Primary immunodeficiency & $38(11.8)$ \\
\hline - Common variable immunodeficiency disorder & $17(5.3)$ \\
\hline - Other primary antibody deficiency & $9(2.8)$ \\
\hline - Specific polysaccharide antibody deficiency & $6(1.9)$ \\
\hline - X-linked agammaglobulinemia & $4(1.3)$ \\
\hline - Hyper lgM syndrome & $3(0.9)$ \\
\hline - Undefined combined immunodeficiency & $12(3.8)$ \\
\hline - Thymoma with immunodeficiency & $62(19.3)$ \\
\hline - Other & $18(5.6)$ \\
\hline Secondary immunodeficiency & $10(3.1)$ \\
\hline - Haematological cause & $2(0.6)$ \\
\hline \multicolumn{2}{|l|}{ - Rheumatological cause } \\
\hline \multicolumn{2}{|l|}{ - Other cause } \\
\hline \multicolumn{2}{|l|}{ Unknown } \\
\hline Immunoglobulin product & $167(52.2)$ \\
\hline - Intravenous immunoglobulin & $133(41.6)$ \\
\hline - Subcutaneous immunoglobulin & $16(5.0)$ \\
\hline - Prophylactic antibiotics only & $4(1.3)$ \\
\hline \multicolumn{2}{|l|}{ - Unknown } \\
\hline Immunoglobulin level & $3.65(1.73-4.92)$ \\
\hline Pre-treatment lgG (g/L) & $9.46(8.20-11.06)$ \\
\hline Trough IgG (g/L) & $0.16(0.05-0.61)$ \\
\hline $\lg A(g / L)$ & $0.3(0.10-0.68)$ \\
\hline \multicolumn{2}{|l|}{$\lg M(g / L)$} \\
\hline SARS-CoV-2 PCR status at study entry & $1(0.31)$ \\
\hline Positive & $283(88.4)$ \\
\hline Negative & $36(11.3)$ \\
\hline Unknown & \\
\hline
\end{tabular}

Eighteen participants ( $n=18 / 320,5.6 \%$ ) had suffered PCR-proven SARS-CoV-2 infection prior to study entry; these participants were significantly younger (52.0 vs. 59.0 years, $p=0.02$ ) than individuals who remained SARS-CoV-2 infection-naive (Table 2). Only one participant remained SARS-CoV-2 PCR positive on study entry. Eleven participants $(n=11 / 18,61.1 \%)$ returned negative nasopharyngeal swabs at the time of study entry and six participants declined further investigation. No specific immunological characteristics defined the population with apparent viral clearance: 4 patients had CVID, 2 other primary antibody deficiencies, 1 Good's syndrome, 1 XLA and 3 secondary immunodeficiencies; $54.4 \%(n=6 / 11)$ made no persistent serological response to infection and $36.4 \%$ 
$(n=4 / 11)$ no serological response to subsequent vaccination. T cell responses were assessed in five of the six seronegative individuals by interferon-gamma ELISPOT: $100 \%\left(n=5 / 5\right.$, median spots $/ 10^{6}$ cell $\left.=158\right)$ mounted responses against spike peptide pools and $60 \%\left(n=3 / 5\right.$, median spots $/ 10^{6}$ cells $\left.=45\right)$ to the nucleocapsid peptide pools demonstrating $\mathrm{T}$ cell immunity may compensate for the absence of humoral immunity under some circumstances.

Table 2

Comparison of participants in Arm 1 vs Arm 2 of the COV-AD study

\begin{tabular}{|llll|}
\hline & Arm 1 (Prior PCR+ SARS-CoV-2 infection) & Arm 2 (No known PCR+ SARS-CoV-2 infection) & p \\
\hline N & 18 & 302 & - \\
\hline Age (yr) & 52.0 & 59.0 & 0.02 \\
& $(30.3-61.5)$ & $(44.0-69.0)$ & NS \\
\hline Sex (male) $n, \%$ & $7(38.9)$ & $121(40.3)$ & \\
\hline
\end{tabular}

One hundred and sixty-eight participants were sampled one to two months after their second vaccine dose using venous or DBS collection. The overall seropositivity following vaccination in this cohort was $54.8 \%(n=92 / 168)$ and the median IgGAM ratio of seropositive individuals was 2.81 (positive defined as ratio $>1.0$ ), with comparable results in groups sampled by DBS and venous blood (Figure 1A). By comparison, overall seropositivity in 205 healthy participants from the COCO study was $100.0 \%$ with a median IgGAM ratio of 5.51 . There was no significant difference in the percentage of individuals who were seropositive, or the magnitude of the antibody response between participants who had previously had SARS-CoV-2 infection and those who were infection naive (Figure 1B). The Pfizer BioNTech 162b vaccine was associated with significantly greater seroconversion (65.7\% vs $48.0 \%, \mathrm{p}=0.03)$ and antibody responses (IgGAM ratio 3.73 vs 2.39, p=0.0003) in comparison to the AstraZeneca ChAdOx1 nCoV-19 vaccine (Figure 1C). Serological responses from both vaccines display signifiant waning over time (2-way ANOVA, $p=0.001$ ) but recipients of the Pfizer BioNTech vaccine displayed better preservation of antibody responses (2-way ANOVA, p<0.0001) (Figure 1D). Age did not significantly affect the magnitude of antibody responses or seroconversion following vaccination (Figure 1E). Humoral responses following vaccination were variable amongst participants with a range of immunodeficiencies (Figure 1F). As expected, serological responses were not detected in patients with X-linked agammaglobulinaemia (XLA), however $52.2 \%$ of individuals with common variable immunodeficiency mounted a serological response to vaccination. Seropositivity was $75.0 \%$ in individuals with other primary antibody deficiencies (excluding XLA and CVID) and $100.0 \%$ in individuals with specific polysaccharide antibody deficiency. Variability was also observed amongst individuals with secondary immunodeficiencies regardless of aetiology. Thirty-one participants were sampled before and after their second immunisation permitting comparison of pre and post vaccine responses (Figure 1G): seropositivity increased from $29.0 \%$ following the first dose to $61.2 \%$ following the second dose; both vaccines increased the magnitude of the antibody response.

T cell responses to vaccination were studied in 92 individuals following their second vaccine dose (Figure $2 \mathrm{~A}$ ). In responses to a peptide pool derived from the SARS-CoV-2 spike protein, $46.2 \%$ of participants $(n=42 / 91)$ mounted a positive T cell response and a further $11.0 \%$ ( $n=10 / 91)$ mounted a borderline response, as defined by this assay. In response to the SARS-CoV-2 nucleocapsid peptide pool, $8.8 \%(n=8 / 91)$ of participants demonstrated a detectable T cell response and $1.1 \%(n=1 / 91)$ mounted a borderline response. Individuals who had suffered previous PCR+ SARS-CoV-2 infection mounted a significantly greater postvaccination $T$ cell response to the spike protein than those who were infection naive; no significant difference was observed for the nucleocapsid protein (Figure 2A). T cell responses directed towards the spike protein were comparable between the Pfizer BioNTech 162b and AstraZeneca ChAdOx1 nCoV-19 following the second dose of either vaccine (Figure 2B) and persisted as time passed following vaccination (Figure 2C). Participant age did not significantly influence the percentage of participants mounting a $T$ cell response to the spike protein; a trend was observed towards greater magnitude responses in younger participants (Figure 2D). 57.9\% of participants with common variable immunodeficiency disorder mounted a T cell response to the SARS-CoV-2 spike protein following vaccination with a wide range of responses detected in other primary and secondary immunodeficiencies (Figure 2E). Both the Pfizer and AstraZeneca vaccines induced incremental T cell responses following the second vaccine doses in the majority of participants (Figure $2 \mathrm{~F}$ ). A detectable $\mathrm{T}$ cell response was associated with significantly greater seropositivity following vaccination $(79.5 \% \mathrm{vs} 53.8 \%, \mathrm{p}=0.009)$ and antibody responses of significantly greater magnitude (IgGAM ratio 3.08 vs. $2.14, \mathrm{p}=0.03$ ) (Figure $2 \mathrm{G}$ ).

Participants that were seropositive post-vaccination had significantly greater serum IgM concentrations (Figure 3A) and significantly larger numbers of CD19+ peripheral B cells (Figure 3B) compared to those who were seronegative. There was no direct relationship between CD19 B cell numbers and IgM concentration $\left(r^{2}=0.001, p=0.53\right)$. Serum concentrations of both $\lg A$ and $\lg M$ were positively correlated with the magnitude of the antibody response following vaccination (Supplementary Figure 1).

The functionality of antibodies was studied using in vitro, live virus neutralisation assays. Only $37 \%$ of participants with CVID ( $p=0.0001$ ) and $16 \%$ with primary antibody deficiency $(\mathrm{p}=0.0003$ ) displayed $50 \%$ viral neutralising activity or greater, compared to $100 \%$ of healthy controls (Figure $4 \mathrm{~A})$. Neutralising capacity was not significantly impacted by prior SARS-CoV-2 infection status (Figure 4B), type of vaccination received (Figure 4C) or by participants' age (Figure 4D). The capacity of vaccine induced anti-spike IgG antibodies to bind the SARS-CoV-2 delta variant was significantly reduced compared to original Victoria strain $\left(\mathrm{OD}_{450} 1.26\right.$ vs $\left.1.41, \mathrm{p}<0.0001\right)$. $39.4 \%$ of individuals with detectable IgG responses against the Victoria strain fell below the threshold for positivity when the delta variant was substituted into the ELISA assay (Figure 4E).

Ten vaccine-breakthrough, PCR-proven infections have occurred this cohort to date; a further individual was infected between their first and second vaccine dose on the background of prior COVID-19 (Table 3). Eight participants reported new symptoms associated with acute COVID-19 above and beyond any chronic symptoms secondary to their immunodeficiency. $90.0 \%(n=9 / 10)$ of vaccine-breakthrough infections occurred in recipients of the AstraZeneca vaccine at a median interval of 120 days post second-dose and $70.0 \%$ occurred in individual who made no detectable humoral response to vaccination. One participant with secondary immunodeficiency that failed to mount a detectable antibody or cellular response to vaccination, died of COVID-19 
Table 3

Vaccine breakthrough infections in COV-AD participants

\begin{tabular}{|c|c|c|c|c|c|c|c|c|c|c|c|c|}
\hline Patient & Age & Sex & Diagnosis & $\begin{array}{l}\text { Prior } \\
\text { COVID }\end{array}$ & Symptoms & $\begin{array}{l}\text { Duration } \\
\text { of } \\
\text { positivity } \\
\text { (d) }\end{array}$ & $\begin{array}{l}\text { Time } \\
\text { from } \\
\text { second } \\
\text { vaccine } \\
\text { dose to } \\
\text { infection } \\
\text { (d) }\end{array}$ & Vaccine & $\begin{array}{l}\text { Seropositive } \\
\text { post } \\
\text { vaccination }\end{array}$ & $\begin{array}{l}\text { IgGAM } \\
\text { Ratio }\end{array}$ & $\begin{array}{l}\text { B cell count } \\
\left(\times 10^{9} / \mathrm{L}\right)\end{array}$ & Outcome \\
\hline 1 & 54 & $F$ & Undefined CID & $Y$ & $\mathrm{Y}$ & $\begin{array}{l}\text { Not } \\
\text { known - } \\
\text { declined } \\
\text { swabs }\end{array}$ & $\begin{array}{l}1 \text { dose } \\
\text { only }\end{array}$ & $A Z$ & $\mathrm{Y}$ & 5.41 & 4.59 & Alive \\
\hline 2 & 68 & $\mathrm{~F}$ & $\begin{array}{l}\text { Secondary - } \\
\text { rheumatology }\end{array}$ & $\mathrm{N}$ & $\mathrm{Y}$ & 29 & 94 & $A Z$ & $\mathrm{~N}$ & 0.25 & Undetectable & Deceased \\
\hline 3 & 38 & M & CVID & $N$ & Y & 28 & 116 & $A Z$ & Y & 2.47 & 0.17 & Alive \\
\hline 4 & 38 & $\mathrm{~F}$ & $\begin{array}{l}\text { Primary } \\
\text { antibody } \\
\text { deficiency }\end{array}$ & $\mathrm{N}$ & $\mathrm{Y}$ & 22 & 143 & $A Z$ & $\mathrm{~N}$ & 0.63 & $n / a$ & Alive \\
\hline 5 & 37 & $\mathrm{~F}$ & CVID & $\mathrm{N}$ & $\mathrm{Y}$ & $\begin{array}{l}\text { Not } \\
\text { known - } \\
\text { declined } \\
\text { swabs }\end{array}$ & 138 & $A Z$ & $\mathrm{~N}$ & 0.27 & 0.01 & Alive \\
\hline 6 & 60 & M & CVID & $N$ & U & $80^{*}$ & 92 & Pfizer & $N$ & 0.19 & 0.03 & Alive \\
\hline 7 & 61 & $\mathrm{~F}$ & $\begin{array}{l}\text { Secondary - } \\
\text { heamatological }\end{array}$ & $\mathrm{N}$ & U & $11^{\$}$ & 181 & $A Z$ & $\mathrm{Y}$ & 1.59 & 0.13 & Alive \\
\hline 8 & 45 & $\mathrm{~F}$ & CVID & $\mathrm{N}$ & $\mathrm{Y}$ & 63 & 61 & $A Z$ & $\mathrm{~N}$ & 0.18 & 0.01 & Alive \\
\hline 9 & 44 & M & CVID & $\mathrm{N}$ & $\mathrm{Y}$ & 16 & 111 & $A Z$ & $\mathrm{~N}$ & 0.48 & 0.01 & Alive \\
\hline 10 & 50 & $\mathrm{~F}$ & $\begin{array}{l}\text { Secondary - } \\
\text { heamatological }\end{array}$ & $\mathrm{N}$ & $\mathrm{Y}$ & $24^{\$}$ & 199 & $A Z$ & $\mathrm{~N}$ & 0.62 & 0.45 & Alive \\
\hline 11 & 61 & $M$ & CVID & $N$ & $\mathrm{U}$ & 10 & 120 & $A Z$ & $Y$ & 2.26 & 0.24 & Alive \\
\hline
\end{tabular}

\section{Discussion}

Understanding the immunogenicity and efficacy of vaccinations is essential to guide global vaccination strategies and when to deploy non-pharmacological countermeasures to protect the immunologically vulnerable $[19,20]$. Herein, we report the immunogenicity of the AstraZenca ChAdOx1 nCoV-19 and Pfizer BioNTech $162 \mathrm{~b}$ vaccinations in patients with antibody deficiency, a cohort who have historically responded poorly to vaccinations [24-26].

Overall seropositivity following vaccination was $54.8 \%$, significantly lower than healthy controls; comparable seropositivity was observed in the two largest subgroups of patients, common variable immunodeficiency (52.1\%) and secondary immunodeficiency arising from haematological cause (55.8\%). However, less than $10 \%$ of individuals with primary or secondary antibody deficiency made a neutralising antibody response equivalent to that of healthy controls following two doses of a SARS-CoV-2 vaccine. Furthermore, almost $40 \%$ of vaccine responders did not demonstrate binding to the SARS-CoV- 2 delta variant and are likely to have limited neutralising capacity against it [27]. These data suggest that vaccine-induced antibody responses are inadequate in the majority of individuals with antibody deficiency and additional strategies such as the use of prophylactic monoclonal antibodies to provide passive protection and antivirals are likely to be necessary to prevent severe disease.

T cell responses to vaccination displayed significant heterogeneity in this cohort, as has been found in similar studies using identical laboratory methods [28]. The interferon-gamma release assay was originally validated to study T cell responses following natural infection, where it displays $98 \%$ sensitivity [29]. 46.2\% of participants in COVAD mounted a detectable T cell response following vaccination using this assay, compared to $54 \%$ of healthy individuals [30]. T cell responses in patients with evidence of previous SARS-CoV-2 infection were significantly greater than those detected following vaccination in infection-naive participants. The discordance between the detection of vaccine- and infection-induced $\mathrm{T}$ cell responses potentially arises from differences in MHC restriction to the constituents of the peptide pool and the antigen-specific $\mathrm{T}$ cell repertoire in each circumstance.

Our study found strong T cell responses in XLA patients, as has been shown previously [5]. The clinical correlates of infection- or vaccine-induced T cell responses in patients with antibody deficiency, in particular, in the absence of humoral immunity remain uncertain. The absence of humoral immunity is a characteristic feature of individuals with prolonged SARS-CoV-2 infection [7]; however, robust T cell responses can limit the severity of disease in some individuals in the absence of humoral immunity as has been shown previously in patients with haematological malignancy [31]. Further studies are necessary to characterise the quality and breadth of $\mathrm{T}$ cell responses and its relationship to the development of effective humoral immunity following infection and vaccination in more detail. 
With respect to vaccination strategies, we have shown that the Pfizer BioNTech 162b vaccine demonstrated significantly greater humoral immunogenicity in patients with antibody deficiency than the AstraZeneca ChAdOx1 nCoV-19 vaccination, a finding consistent with larger studies in healthy individuals [13, 32] and renal transplant recipients [28]. Furthermore, over $90 \%$ of vaccine breakthrough infections occurred in recipients of the AstraZeneca vaccine, $60.0 \%$ of whom made no serological response to the initial 2-dose vaccine schedule. Studies in the general population have suggested adenoviral-vectored vaccines demonstrate reduced vaccine-efficacy against severe disease when directly compared to mRNA vaccines

(https://www.cdc.gov/mmwr/volumes/70/wr/mm7038e1.htm). These observations support the use of mRNA vaccines in patients with antibody deficiency.

It could be argued that the deployment of a 3rd dose of vaccination in individuals that have not responded to a first dose is futile. However, we have found that serological and cellular responses to the SARS-CoV-2 spike protein were positively incremented by the second vaccine dose, in keeping with previous studies in patients with inborn errors of immunity [16] suggesting potential benefit from further doses. Further studies will be necessary to explore whether different vaccination combinations (homologous/heterologous) or dosing schedules may improve responses and efficacy in patients with primary and secondary humoral immunodeficiencies.

Existing studies have reported wide variation in the serological response to vaccination in patients with immunodeficiency: post-vaccine seroprevalence have ranged from 20.0-80.0\% [14-18]. The COVAD study is the largest reported study of patients with antibody deficiency and finds a seropositivity rate of $54.8 \%$ overall. At a cohort level, total B cell numbers were the principal determinate of serological response to vaccination, also in keeping with other SARS-CoV-2 vaccine studies [17]. Additional correlates of vaccine responsiveness remain to be elucidated: Salinas et al demonstrated patients with CVID have a relative paucity of receptor-binding domain-specific, $\mathrm{CD} 19^{+} \mathrm{CD} 24^{+} \mathrm{CD} 27^{+} \mathrm{B}$ cells compared to healthy controls [18] and Hagin et a/ were unable to demonstrate a common T-cell immunophenotype in vaccine non-responders beyond an inverted CD4/CD8 ratio [17]. Future work in COV-AD will employ detailed phenotypic and functional profiling to investigate potential correlates of vaccine immunogenicity and efficacy within this heterogeneous cohort.

This is a large study in a rare disease cohort and, although heterogeneous, we have had the opportunity to compare the immunogenicity of an mRNA and adenoviral-vectored vaccine in an immunodeficient cohort. To some extent, the generalisability of our study to the wider world is confounded by the extended UK vaccine schedule, which has not yet been widely adopted elsewhere. On the one hand, extension of the interval between first and second doses has been associated with greater neutralising antibody responses and enrichment of virus specific CD4+ T cells in healthy individuals [33], but shorter dosing intervals were associated with better humoral responses in a smaller study of patients vaccinated following treatment with B cell depleting agents (Shields AM, in press). There is an urgent need for further studies that explore how to maximise vaccine immunogenicity and efficacy in larger and heterogeneous cohorts of immune deficient patients.

In conclusion, we demonstrate profound impairment of serological responses following SARS-CoV-2 vaccination in patients with antibody deficiency and evidence of the superior immunogenicity of the Pfizer BioNTech 162b vaccine. These data highlight the ongoing risk of SARS-CoV-2 infection in antibody deficiency patients and should inform public health policy on vaccination strategies and other treatments to prevent morbidity and mortality.

\section{Abbreviations}

\begin{tabular}{|ll|}
\hline APDS-1 & Activated PI3K delta syndrome 1 \\
\hline CID & Combined immunodeficiency \\
\hline COVID-19 & Coronavirus disease 2019 \\
\hline CTLA-4 & Cytotoxic T-lymphocyte-associated protein 4 \\
\hline CVID & Common variable immunodeficiency disorder \\
\hline DBS & Dried blood spot \\
\hline ELISA & Enzyme-linked immunosorbent assay \\
\hline NFKB2 & Nuclear Factor Kappa B Subunit 2 \\
\hline PAD & Primary antibody deficiency \\
\hline PCR & Polymerase chain reaction \\
\hline SAMD9L & Sterile Alpha Motif Domain Containing 9 Like \\
\hline SARS-CoV-2 & Severe acute respiratory syndrome coronavirus 2 \\
\hline SID & Secondary immunodeficiency \\
\hline SPAD & Specific polysaccharide antibody deficiency \\
\hline XLA & X-linked agammaglobulinaemia \\
\hline
\end{tabular}

\section{Declarations}

\section{Acknowledgements}

The authors are grateful to the staff of the University of Birmingham Clinical Immunology Service for facilitating sample processing for this study, the Saving Lives charity (Registered Charity 1144855) for facilitating remote sampling of study participants, The Binding Site Ltd for their ongoing support of the 
University of Birmingham COVID-19 research programme and Oxford Immunotec for their assistance with the T cell assays.

Role of the funding source: This study was funded by United Kingdom Research and Innovation (MR/W002663/1)

Competing interests: The authors declare no competing interests.

Data availability:

The datasets generated during and/or analysed during the current study are available from the corresponding author on reasonable request

\section{Code availability}

Not applicable

Author contributions: AMS, SOB and AGR designed and supervised the study. SEF, HJH, SAT, CT, FA, ZS undertook experimental work and analysis for the study. SW, FM, NV, SG, SJ, AH, CB, SE, DML, SYP, SS, AMS, SOB and AGR recruited patients to the study and acted as local site principal investigators. HW, GH, NC provided administrative and database support for the study and facilitated patient recruitment to the study. PK, SOB and AGR provided senior leadership and strategic oversite for the study. AMS analysed the data, wrote the first draft of the manuscript and revised the manuscript. All authors contributed the revision of the manuscript and read and approved the final version.

\section{Ethical approval}

This study was approved by the London - Dulwich Research Ethics Committee (REC reference: 21/LO/0162) and funded by United Kingdom Research and Innovation (MR/W002663/1). Serological responses from healthy individuals are from participants recruited to the COVID-19 Convalescent (COCO) immunity study (REC reference 20/HRA/1817). All participants provided written informed consent prior to participation in this study.

\section{Consent to participate}

All participants provided written informed consent prior to participation in the COV-AD study.

\section{Consent for publication}

Not applicable

\section{COV-AD Consortium}

Zahra Ahmed ${ }^{1}$

Hollie Bancroft ${ }^{2}$

Michelle Bates ${ }^{2}$

Hayley Clifford ${ }^{2}$

Georgina Davis ${ }^{3}$

Joanne Dasgin ${ }^{1}$

Mohammad Dinally ${ }^{1}$

Fatima Dhalla ${ }^{4}$

Elena Efstathiou ${ }^{1}$

Mark Gompels ${ }^{5}$

Dan Hartland ${ }^{6}$

Madeeha Hoque ${ }^{1}$

Emily Heritage $^{7}$

Deborah Hughes ${ }^{8}$

Ann Ivory ${ }^{8}$

Rashmi Jain ${ }^{4}$

Sinead Kelly ${ }^{9}$ 
Theresa McCarthy ${ }^{1}$

Christopher McGee ${ }^{2}$

Daniel Mullan ${ }^{3}$

Hadeil Morsi ${ }^{4}$

Eileen $\mathrm{O}^{\prime} \mathrm{Grady}^{10}$

Shannon Page ${ }^{1}$

Nicholas Peters ${ }^{4}$

Timothy Plant ${ }^{1}$

Archana Shajidevadas ${ }^{11}$

Malgorzata Slowinsksa ${ }^{5}$

Zehra Suleiman $^{1}$

Neil Townsend ${ }^{1}$

Charlotte Trinham ${ }^{1}$

Stuart Wareham ${ }^{3}$

Sinead Walder ${ }^{1}$

\section{Consortium Affiliations}

1. Clinical Immunology Service, Institute for Immunology and Immunotherapy, University of Birmingham, UK

2. University Hospitals Birmingham NHS Foundation Trust, Birmingham, UK

3. Department of Immunology and Allergy, University Hospital Plymouth NHS Trust, Plymouth, UK

4. Department of Clinical Immunology, Oxford University Hospitals NHS Foundation Trust, Oxford, UK

5. Department of Immunology, North Bristol NHS Trust, Bristol, UK

6. Saving Lives Charity, MIDRU Building, Heartlands Hospital, Birmingham, UK

7. Institute of Translational Medicine, University of Birmingham, Birmingham, UK

8. Department of Immunology, University Hospital North Midlands, Stoke, UK

9. Newcastle Upon Tyne Hospitals NHS Foundation Trust, Newcastle, UK

10. Department of Allergy and Clinical Immunology, Leeds Teaching Hospitals NHS Trust, Leeds, UK

Research and Development Department, University Hospital Plymouth NHS Trust, Plymouth, UK

\section{References}

1. Weinreich DM, et al., REGEN-COV Antibody Combination and Outcomes in Outpatients with Covid-19. N Engl J Med, 2021.

2. Hanrath AT, Payne BAI, Duncan CJA. Prior SARS-CoV-2 infection is associated with protection against symptomatic reinfection. J Infect, 2020.

3. Lumley SF, et al., Antibody Status and Incidence of SARS-CoV-2 Infection in Health Care Workers. N Engl J Med, 2020.

4. Shields AM, et al. COVID-19: Seroprevalence and Vaccine Responses in UK Dental Care Professionals. J Dent Res. 2021;100(11):1220-7.

5. Buckland MS, et al. Treatment of COVID-19 with remdesivir in the absence of humoral immunity: a case report. Nat Commun. 2020;11(1):6385.

6. Bradley RE, et al., Persistent COVID-19 Infection in Wiskott-Aldrich Syndrome Cleared Following Therapeutic Vaccination: a Case Report. J Clin Immunol, 2021: p. 1-4.

7. Brown LK, et al., Treatment of chronic or relapsing COVID-19 in immunodeficiency. J Allergy Clin Immunol, 2021.

8. McKemey E, et al. Resolution of Persistent COVID-19 After Convalescent Plasma in a Patient with B Cell Aplasia. J Clin Immunol. 2021;41(5):926-9.

9. Group RC, et al., Casirivimab and imdevimab in patients admitted to hospital with COVID-19 (RECOVERY): a randomised, controlled, open-label, platform trial. medRxiv, 2021: p. 2021.06.15.21258542.

10. Polack FP, et al. Safety and Efficacy of the BNT162b2 mRNA Covid-19 Vaccine. N Engl J Med. 2020;383(27):2603-15.

11. Baden LR, et al. Efficacy and Safety of the mRNA-1273 SARS-CoV-2 Vaccine. N Engl J Med. 2021;384(5):403-16. 
12. Voysey M, et al. Safety and efficacy of the ChAdOx1 nCoV-19 vaccine (AZD1222) against SARS-CoV-2: an interim analysis of four randomised controlled trials in Brazil, South Africa, and the UK. Lancet. 2021;397(10269):99-111.

13. Khoury DS, et al. Neutralizing antibody levels are highly predictive of immune protection from symptomatic SARS-CoV-2 infection. Nat Med. 2021;27(7):1205-11.

14. Arroyo-Sánchez D, et al., Immunogenicity of Anti-SARS-CoV-2 Vaccines in Common Variable Immunodeficiency. J Clin Immunol, 2021: p. 1-13.

15. Bergman P, et al., Safety and efficacy of the mRNA BNT162b2 vaccine against SARS-CoV-2 in five groups of immunocompromised patients and healthy controls in a prospective open-label clinical trial. medRxiv, 2021: p. 2021.09.07.21263206.

16. Delmonte OM, et al. Antibody responses to the SARS-CoV-2 vaccine in individuals with various inborn errors of immunity. J Allergy Clin Immunol. 2021;148(5):1192-7.

17. Hagin D, et al. Immunogenicity of Pfizer-BioNTech COVID-19 vaccine in patients with inborn errors of immunity. J Allergy Clin Immunol. 2021;148(3):73949.

18. Salinas AF, et al., SARS-CoV-2 Vaccine Induced Atypical Immune Responses in Antibody Defects: Everybody Does their Best. J Clin Immunol, 2021: p. 114.

19. Meyts I, et al., Coronavirus Disease 2019 in patients with inborn errors of immunity: an international study. Journal of Allergy and Clinical Immunology. 20. Shields AM, et al., COVID-19 in patients with primary and secondary immunodeficiency: The United Kingdom experience. J Allergy Clin Immunol, 2020.

21. Bosworth A, et al. Rapid implementation and validation of a cold-chain free SARS-CoV-2 diagnostic testing workflow to support surge capacity. J Clin Virol. 2020;128:104469.

22. Morley GL, et al. Sensitive Detection of SARS-CoV-2-Specific Antibodies in Dried Blood Spot Samples. Emerg Infect Dis. 2020;26(12):2970-3.

23. Cook AM, et al. Validation of a combined ELISA to detect IgG, IgA and IgM antibody responses to SARS-CoV-2 in mild or moderate non-hospitalised patients. J Immunol Methods. 2021;494:113046.

24. Goldacker S, et al. Active vaccination in patients with common variable immunodeficiency (CVID). Clin Immunol. 2007;124(3):294-303.

25. Gardulf A, et al., Predictive markers for humoral influenza vaccine response in patients with common variable immunodeficiency. J Allergy Clin Immunol, 2018. 142(6): p. 1922-1931.e2.

26. Friedmann D, et al. Preserved Cellular Immunity Upon Influenza Vaccination in Most Patients with Common Variable Immunodeficiency. J Allergy Clin Immunol Pract. 2020;8(7):2332-40.e5.

27. Earle KA, et al. Evidence for antibody as a protective correlate for COVID-19 vaccines. Vaccine. 2021;39(32):4423-8.

28. Prendecki $\mathrm{M}$, et al., Comparison of humoral and cellular responses in kidney transplant recipients receiving BNT162b2 and ChAdOx1 SARS-CoV-2 vaccines. medRxiv, 2021: p. 2021.07.09.21260192.

29. Kruse M, et al. Performance of the T-SPOT(®).COVID test for detecting SARS-CoV-2-responsive T cells. Int J Infect Dis. 2021;113:155-61.

30. Lindemann M, et al., Humoral and Cellular Vaccination Responses against SARS-CoV-2 in Hematopoietic Stem Cell Transplant Recipients. Vaccines (Basel), 2021. 9(10).

31. Bange EM, et al. CD8(+) T cells contribute to survival in patients with COVID-19 and hematologic cancer. Nat Med. 2021;27(7):1280-9.

32. van Gils MJ, et al., Four SARS-CoV-2 vaccines induce quantitatively different antibody responses against SARS-CoV-2 variants. medRxiv, 2021: p. 2021.09.27.21264163.

33. Payne RP, et al. Immunogenicity of standard and extended dosing intervals of BNT162b2 mRNA vaccine. Cell. 2021;184(23):5699-714.e11.

\section{Figures}


A

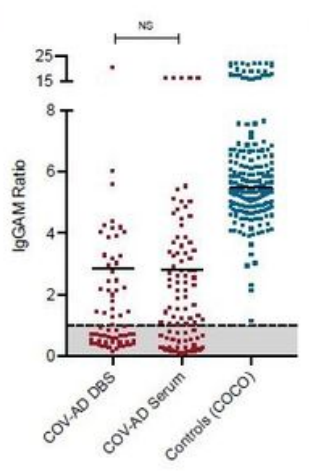

E

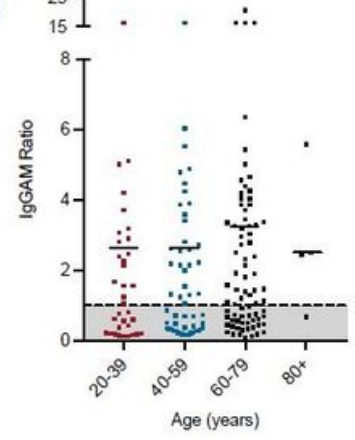

B

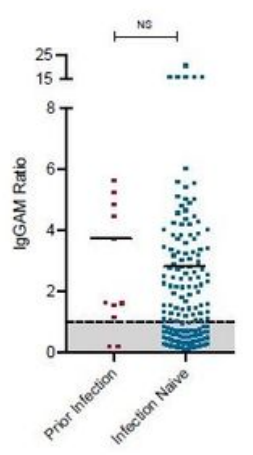

F
C
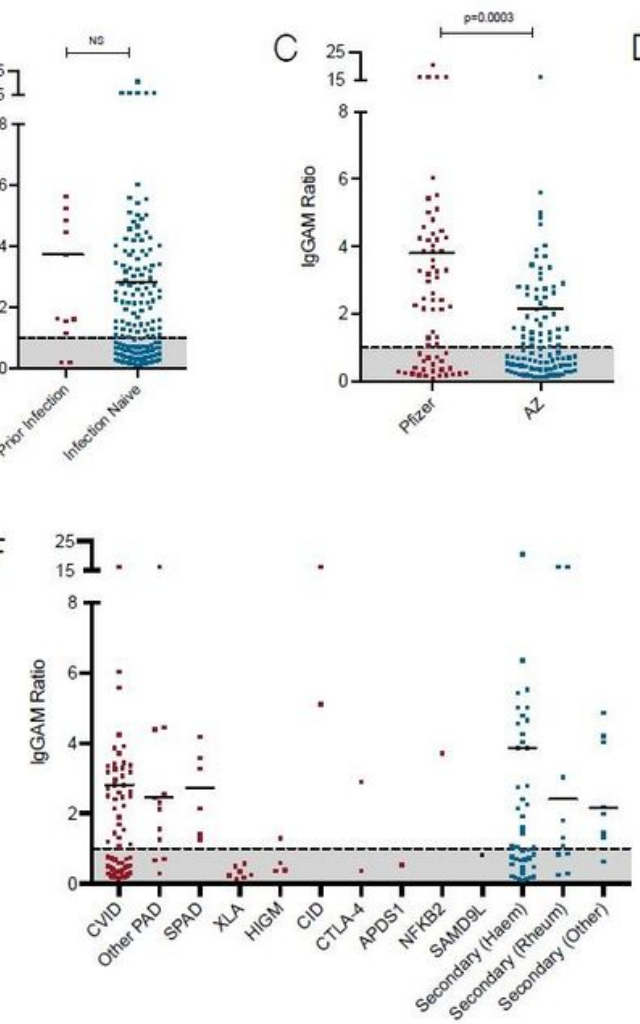

D $\quad{ }_{15}^{25} 7 . .$.

- Pfizer

- $A Z$

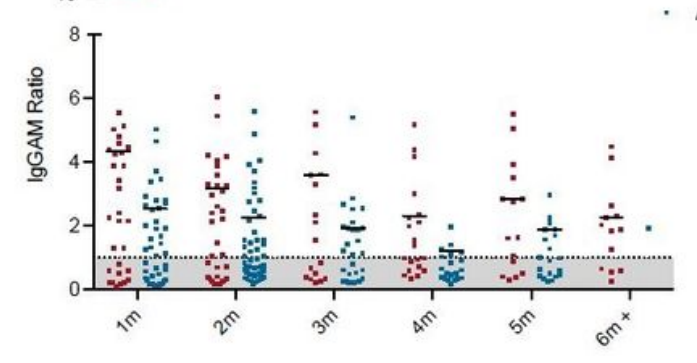

G

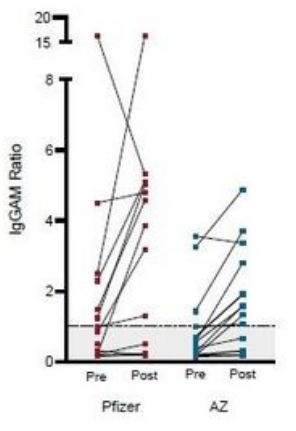

Figure 1

Serological responses to SARS-CoV-2 vaccination in individuals with antibody deficiency. Comparison of serological responses to SARS-CoV-2 vaccination in COV-AD participants. (A) COV-AD participants sampled $1-2 \mathrm{~m}$ post second vaccine dose via dried blood spot (DBS) or serum and in comparison to healthy controls (COCO). (B) Comparison of individuals with prior PCR proven infection and those who were infection naive sampled 1-2m post second vaccine dose. (C) Comparison of recipients of the AstraZeneca ChAdOx1 nCoV-19 and the Pfizer BioNTech 162b vaccines sampled 1-2m post second vaccine dose. (D) Comparison of serological responses over time from vaccination. (E) Comparison of serological responses across age groups in individuals sampled 1-2m post second vaccine dose. (F) Comparison of serological responses by underlying immunodeficiency in participants samples $1-2 \mathrm{~m}$ post second vaccine dose. (G) Dynamic serological response before and after second vaccine dose. Results are presented as the IgGAM ratio with the grey shaded area representing the results below the cut-off for positivity. Horizontal bars represent the median of seropositive results. 

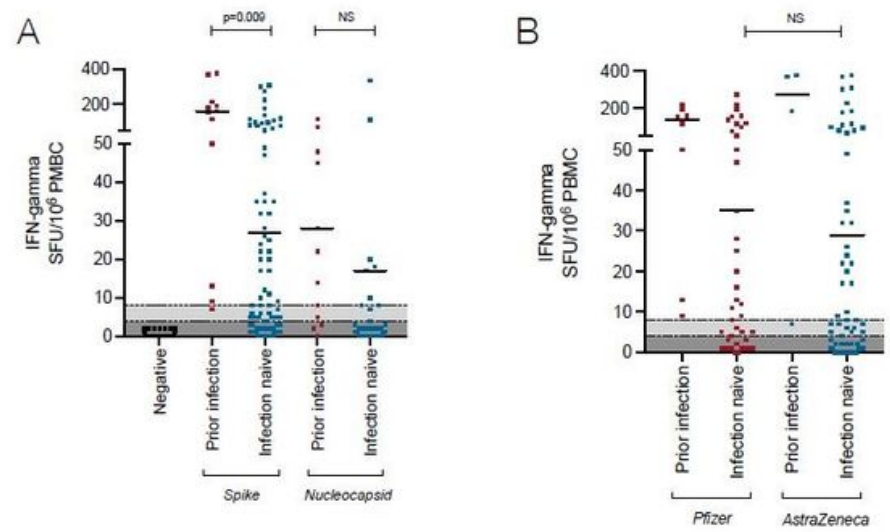
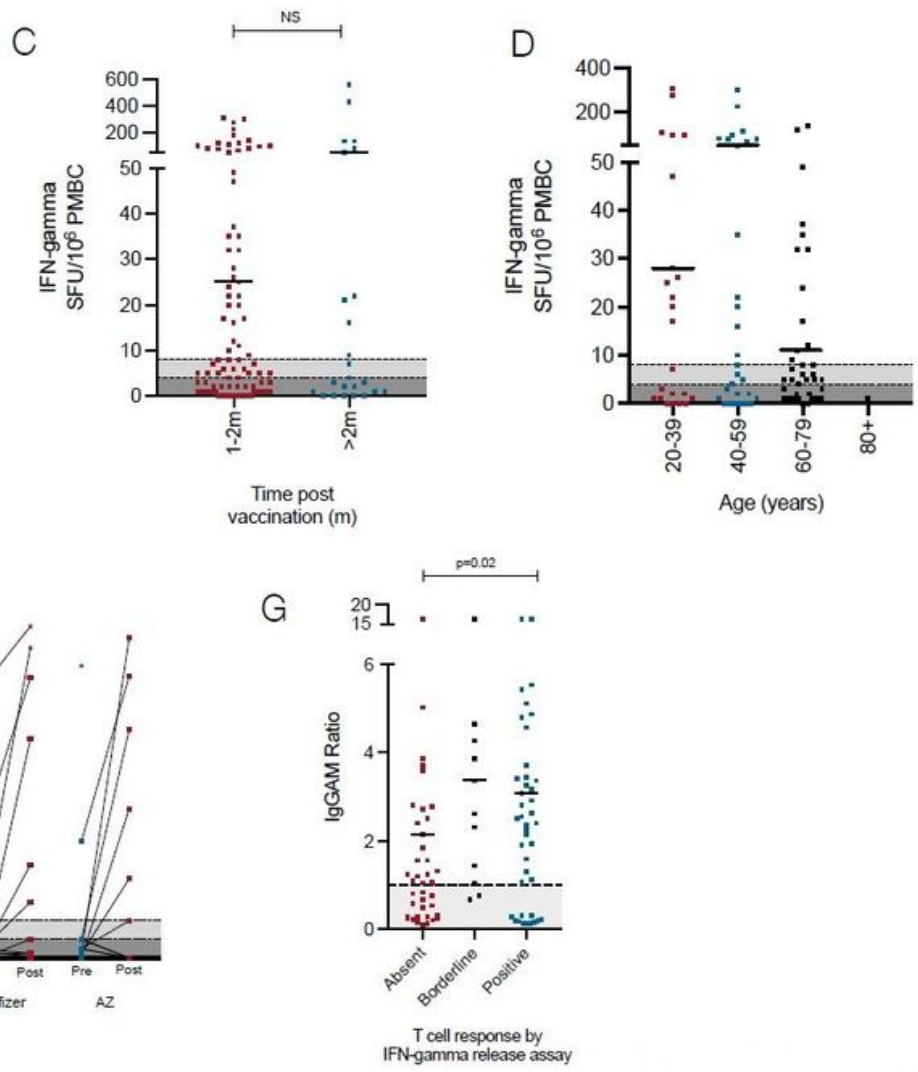

E

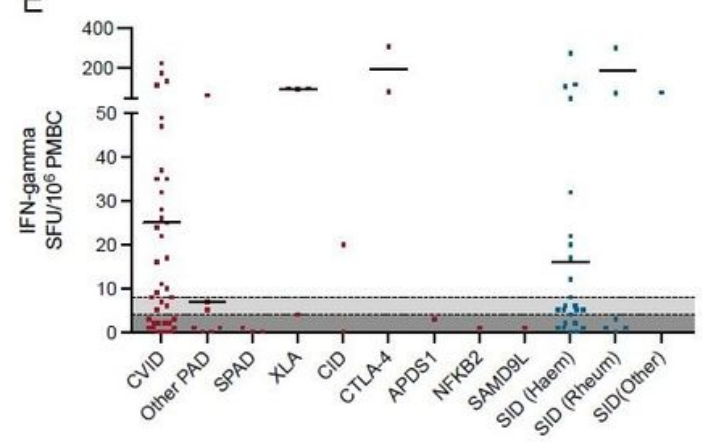

$\mathrm{F}$

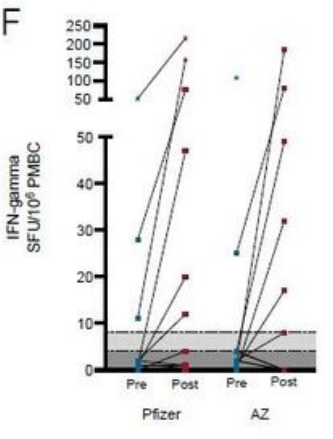

\section{Figure 2}

Cellular responses to SARS-CoV-2 vaccination in individuals with antibody deficiency. Comparison of cellular responses following SARS-CoV-2 vaccination in COV-AD participants using an IFN-gamma release assay. (A) Comparison of T cell responses to spike and nucleocapsid peptide pools in individuals with prior PCR proven SARS-CoV-2 infection and those who were infection naive, in participants sampled 1-2m post second vaccine dose. (B) Comparison of cellular responses to spike peptide pools between the Pfizer and AstraZeneca vaccines in participants sampled 1-2m post second vaccine dose. (C) Comparison of the cellular responses to spike peptide pools over time. (D) Comparison of the cellular responses to spike peptide pools by age in participants sampled 1-2m post second vaccine dose. (E) Comparison of the cellular responses to spike peptide pools by underlying immunodeficiency in participants sampled 1-2m post second vaccine dose. (F). Dynamic changes in cellular response to spike peptide pools before and after the second vaccine dose. (G) Relationship between the $\mathrm{T}$ cell response to spike peptide pools and the magnitude of the anti-spike antibody response in participants sampled $1-2 \mathrm{~m}$ post second vaccine dose. Results are presented as the number of IFN-gamma producing spots per $10^{6}$ cells. Dark grey shaded areas represent no response, light grey shaded areas represent borderline response, as per the manufacturers instructions. 
A

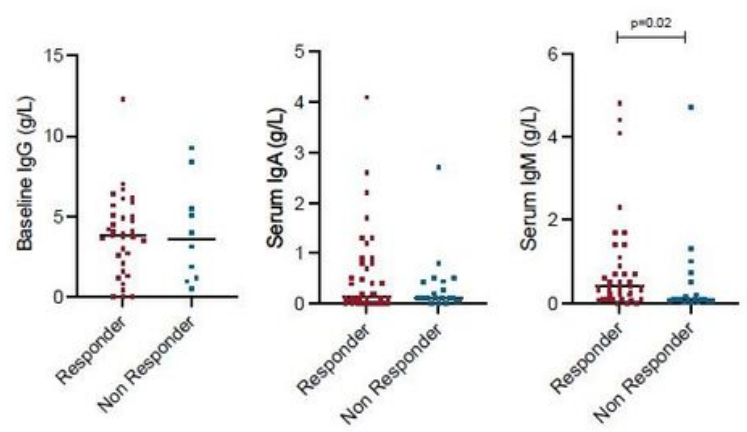

$B$

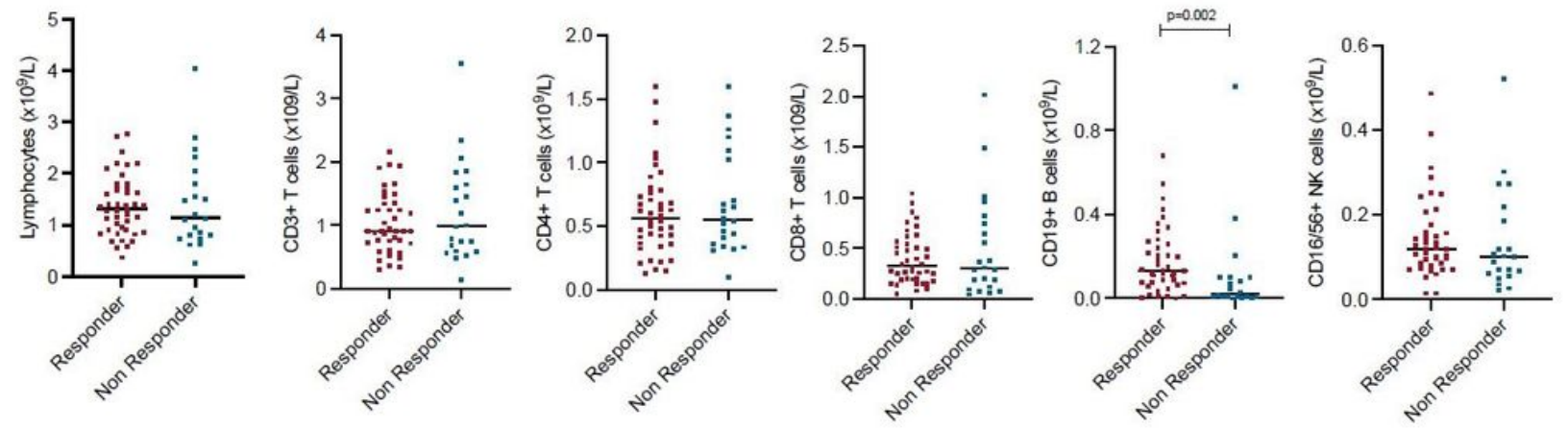

Figure 3

Correlates of seropositivity following SARS-CoV-2 vaccination in individuals with antibody deficiency. Comparison of pre-vaccination immunological parameters between seropositive and seronegative participants sampled 1-2 months following their second vaccine dose: (A) Pre-treatment serum IgG concentration and current serum IgA and IgM concentration. (B) Total lymphocyte count and lymphocyte subset enumeration.

A

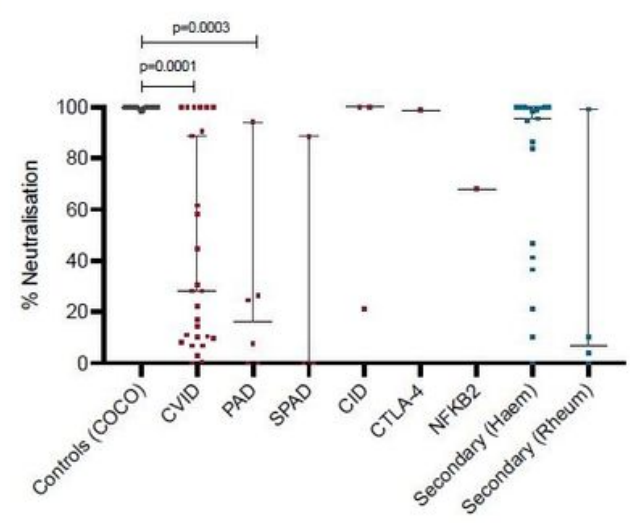

B

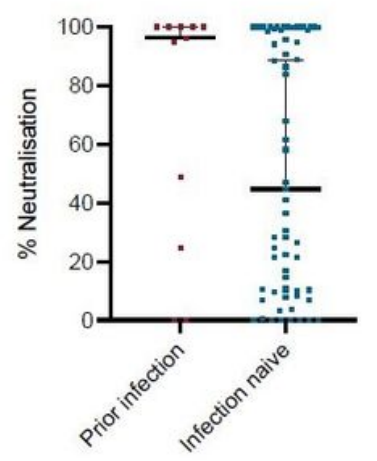

C

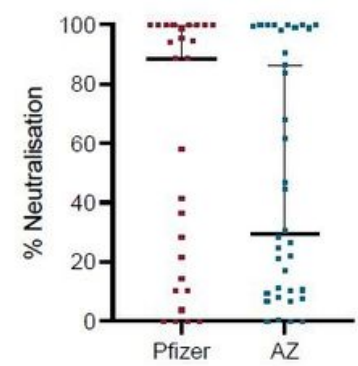

D

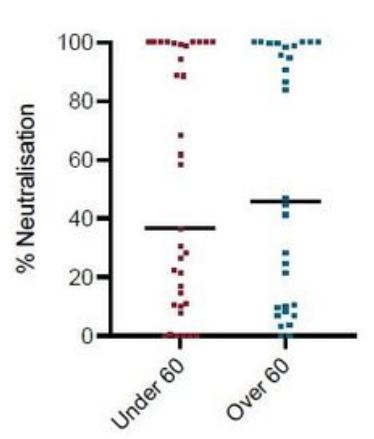

E

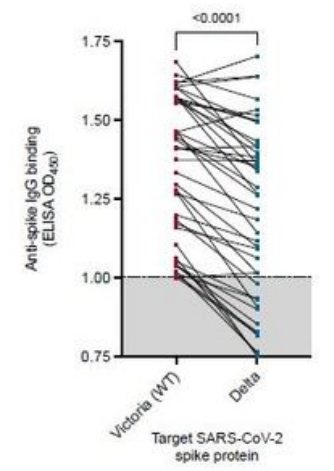


Functional immunity following SARS-CoV-2 vaccination in individuals with antibody deficiency. Serum neutralisation capacity was assessed using live virus neutralisation in seropositive individuals sampled 1-2 months post second vaccine dose. (A) Serum neutralising antibody capacity of seropositive individuals by underlying immunodeficiency. (B) Comparison of serum neutralising antibody capacity between individuals with prior PCR proven SARS-CoV-2 infection and those who were infection naive. (C) Comparison of serum neutralising antibody capacity between recipients of the Pfizer and AstraZeneca vaccinations. (D) Comparison of serum neutralising antibody capacity by age of participants. (E) Comparison of binding of vaccine-induced IgG antibodies from participants sampled 1-2 months post vaccination to the wild-type (Victoria) SARS-CoV-2 spike protein and the Delta variant of concern within an ELISA detection system.

\section{Supplementary Files}

This is a list of supplementary files associated with this preprint. Click to download.

- Sfig1.jpg

- Sfig2.jpg

- SupplementaryMethodsv1.0.docx 